\title{
AFM based dielectric spectroscopy: extended frequency range through excitation of cantilever higher eigenmodes.
}

\author{
Luis A. Miccio ${ }^{1,2,3^{*}}$, Mohammed M. Kummali1 ${ }^{1,3}$, Gustavo A. Schwartz ${ }^{1,2}$, Ángel Alegría ${ }^{1,3}$, \\ Juan Colmenero ${ }^{1,2,3}$ \\ ${ }^{1}$ Centro de Física de Materiales (CSIC-UPV/EHU), P. M. de Lardizabal 5, 20018 San Sebastián, Spain \\ ${ }^{2}$ Donostia International Physics Center, P. M. de Lardizabal 4, 20018 San Sebastián, Spain \\ ${ }^{3}$ Departamento de Física de Materiales (UPV/EHU), 20080 San Sebastián, Spain \\ luisalejandro_miccio@ehu.es \\ *Corresponding author
}

Keywords: AFM, dielectric spectroscopy, structure and dynamics, polymer blends.

\begin{abstract}
In the last years, a new AFM based dielectric spectroscopy approach has been developed for measuring the dielectric relaxation of materials at the nanoscale, the so called nanoDielectric Spectroscopy ( $n \mathrm{DS})$. In spite of the effort done so far, some experimental aspects of this technique remain still unclear. In particular, one of these aspects is the possibility of extending the experimental frequency range, to date limited at high frequencies by the resonance frequency of the AFM cantilever as a main factor. In order to overcome this limitation, the electrical excitation of cantilever higher eigenmodes for measuring the dielectric relaxation is here explored. Thus, in this work we present a detailed experimental analysis of the electrical excitation of the cantilever second eigenmode. Based on this analysis we show that the experimental frequency range of the AFM based dielectric spectroscopy can be extended by nearly two decades with a good signal-to-noise ratio. By using the combination of first and second cantilever eigenmodes we study dissipation processes on well known PVAc based polymeric samples. Both, relaxation spectra and images with molecular dynamics contrast were thus obtained over this broader frequency range.
\end{abstract}




\section{INTRODUCTION}

During the last decades, broadband dielectric spectroscopy (BDS) has shown to be a very useful technique in the study of the molecular dynamics of insulating materials. The huge frequency range achieved $\left(10^{-5}-10^{12} \mathrm{~Hz}\right)$ and the possibility of measuring under different temperature, pressure, and environmental conditions, allows the observation of a large variety of processes with very different time scales. Within this extraordinary experimental window, molecular and collective dipolar fluctuations, charge transport and polarization effects take place, in turn determining the dielectric response of the material under study [1]].

In the last years, the growing interest in nanostructured materials highlighted the need of measurements providing local material properties. Recently, the electric interaction between the tip of an atomic force microscope (AFM) and the material under study was used as the basis to develop a new technique: Local Dielectric Spectroscopy (LDS) [2]. Within this approach, the electrical interaction resulting by applying an AC voltage to a conductive AFM probe is used to reveal information about the relaxation processes within the material. Therefore, this technique combines the capability of sensing the molecular dynamics as a function of the frequency of BDS with the outstanding spatial resolution of AFM. LDS measurements are based on a PLL setup, and detect the force gradient [3-5]. However, a method based on the detection of force was also developed [6-10], the so called nanoDielectric Spectroscopy $(n \mathrm{DS})[\underline{10}]$. Within these approaches either the measured force or force gradient, resulting from the electrical interaction between the tip and the sample under study, are used to obtain information of the dielectric properties of the material.

The electrical interaction between the AFM probe and the sample allows $n \mathrm{DS}$ to probe locally, 20-30 nm [11], the molecular dynamics of nanostructured materials. However, as the measurements are performed through the motion of an AFM cantilever (i.e. a mechanical system with inherent properties), some method derived limitations are found. In particular, the limited frequency range of the technique should be mentioned as one of the most important. Since the three frequency decades achieved during the 
first experiments [2], the experimental frequency range of LDS and $n \mathrm{DS}$ was later increased to four decades (typically from $1 \mathrm{~Hz}$ to $30 \mathrm{kHz}$ ) $[\underline{5}, \underline{10}]$. There is a twofold origin for the frequency range restriction: on the one hand there is a low frequency limit mainly related with a) the time needed to reach a steady state of cantilever oscillations (and therefore to perform a reliable measurement) and b) the thermal drift, which in turn affects the accuracy of the relative tip-sample position. On the other hand, the high frequency limit is imposed by the electronics and more fundamentally by the oscillation amplitude of the AFM cantilever. To date, $\mathrm{nDS}$ experiments were limited to excitation frequencies lower than the fundamental mode. The fundamental mode is the first of the so called normal modes (or eigenmodes), which in turn are described by the continuous cantilever beam model [12]. In other words, $n \mathrm{DS}$ currently presents the possibility of measuring locally through the AFM spatial resolution, at the expense of a high frequency limit mainly imposed by the cantilever characteristics.

The use of higher eigenmodes for mechanical AFM applications has been widely studied in the last years, and it is commonly known as bimodal atomic force microscopy $[13, \underline{14}]$. Within this approach, the cantilever is simultaneously excited with two driving forces at frequencies matching the first and second eigenmodes [15-17]. Bimodal AFM was also employed for exploring surface potentials in Kelvin probe microscopy, where the electrostatic forces appear at the second eigenmode frequency $[\underline{18}, \underline{19}]$.

In this work we demonstrate the possibility of extending the current AFM based dielectric spectroscopy frequency range by using the second eigenmode of the cantilever. In a first step, we study the electrical excitation (and detection) of the second eigenmode oscillations of a conductive cantilever. In a second step, we analyze the accuracy of the electrical measurements in this extended frequency range by using a dissipation-free sample. Finally, we study a dielectric relaxation process on well known polymeric samples as proof of concept experiments. In this way, here we present for the first time AFM based Dielectric Spectroscopy measurements with an extended experimental frequency window. 


\section{1 nano-Dielectric Imaging \& Spectroscopy Background}

The electric force microscopy $(\mathrm{EFM})$ is based on the electrical force $\left(F_{e}\right)$ resulting from the interaction of a conductive AFM probe with polarizable entities in the material $[\underline{6}$, 20-26] $F_{e}$ can be evaluated by modeling the tip-sample system as a capacitor (capacitance $C$ ): when a voltage $(V)$ is applied, the resulting electrostatic potential energy is $W=1 / 2 C V^{2}$ and the corresponding electrostatic force acting on the tip $F_{e}=$ $d W / d z$ (being $\mathrm{z}$ the coordinate along the tip sample distance). Consequently, when a sinusoidal voltage of frequency $f_{e}=\omega_{e} / 2 \pi$ is applied to the AFM tip $\left[V(t)=V_{0} \sin \left(\omega_{e} t\right)\right]$ the corresponding force is a sinusoidal function (see equation 1) with a component at a frequency double than that used in the excitation due to the quadratic relationship between $F_{e}$ and $V$ (see equation 2 ).

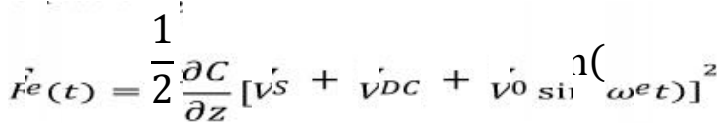

$$
\begin{aligned}
& F^{2} \omega e(t)=-\frac{1}{4} \frac{\partial C}{\partial z} \nu^{2} e^{2}{ }^{c c} \varepsilon^{i}\left(z^{\prime} \omega e t\right)
\end{aligned}
$$

where $V_{S}$ is the surface potential and $V_{D C}$ is the applied DC voltage (if any) [10]. The force amplitude depends both on the experimental conditions and the dielectric properties of the material under investigation. Therefore, by detecting the tip motion component at the second harmonic of the AC excitation voltage, local information about the dielectric relaxation of the materials under investigation can be obtained. This method is known as nano-Dielectric Spectroscopy and requires analyzing the signal from the AFM photodiode with an external Lock-In Amplifier (LIA) in order to obtain both the amplitude and electric phase of the cantilever oscillations. In the case of a loss-free dielectric material the signal amplitude brings information on the static dielectric permittivity, which is frequency independent. However, when dielectric relaxation takes place in the material, the dielectric permittivity becomes frequency 
dependent and a corresponding dielectric loss process appears (which in turn results in a complex capacitance: $\left.C^{*}=C_{0}\left(\omega_{e}\right) \cdot e^{-i \theta\left(\omega_{e}\right)}\right)$ and equation 2 becomes equation 3 .

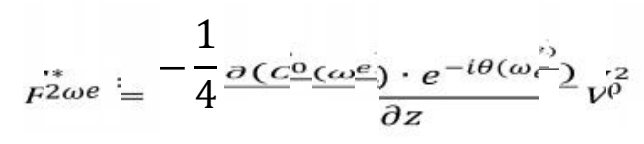

This approach allows collecting information of the frequency dependant dielectric response, either as a) images (nano-Dielectric Imaging, $n \mathrm{DI}$ ) at a fixed frequency or b) frequency spectra (nano-Dielectric Spectroscopy, $n \mathrm{DS}$ ) at a single location. Figure 1 shows a scheme of the implementation of these two experimental methods.

\section{2 nano-Dielectric Imaging}

In this mode, the electrical excitation frequency is fixed and the dielectric interaction between the tip and the sample is mapped by using the single pass method (SP). In SP, both the topography and dielectric contrast of the sample are obtained during the main scan itself. Within this experiment, the sinusoidal voltage is applied to the tip during the main scan and the cantilever response is filtered by the external LIA. Electric phase $(\theta)$ and root mean square oscillation amplitude $\left(\left|R M S_{\text {amp }}\right|\right.$, which is proportional

to $F_{2 \omega_{e}}$ ) of the cantilever signal thus obtained are mapped along with the topography and the mechanical phase (analyzed in the AFM internal LIA). Thus, it is possible to obtain an image with molecular dynamics contrast at fixed frequency and temperature [요, 27]].

\section{3 nano-Dielectric Spectroscopy}

In contrast with the previous method, in this case the experiment is performed at a single location by using the double-pass method (DP). In a first step, the tip-sample distance (or sample topography) is established precisely by a standard 'Tapping' (intermittent contact) experiment. Subsequently, the mechanical cantilever oscillation 
amplitude is set to zero, in order to maintain a constant tip-sample distance (lift mode), and the probe motion generated by the application of an alternating voltage is analyzed. The advantage of the DP method for spectra acquisition resides in avoiding any non-linear interactions produced by larger oscillation amplitudes in piezo driven cantilevers (like in SP). $\left|R M S_{a m p}\right|$ and $\theta$ are simultaneously obtained from the LIA. A reference experiment on a dissipation free sample is performed for each probe in order to establish the zero electric phase accurately (by removing phase shifts associated with the electronics and the mechanical characteristics of the AFM probe). Subsequently, phase shift $(\Delta \theta)$ as a function of the electrical excitation frequency, spectra, are obtained by subtracting this reference from the sample response.
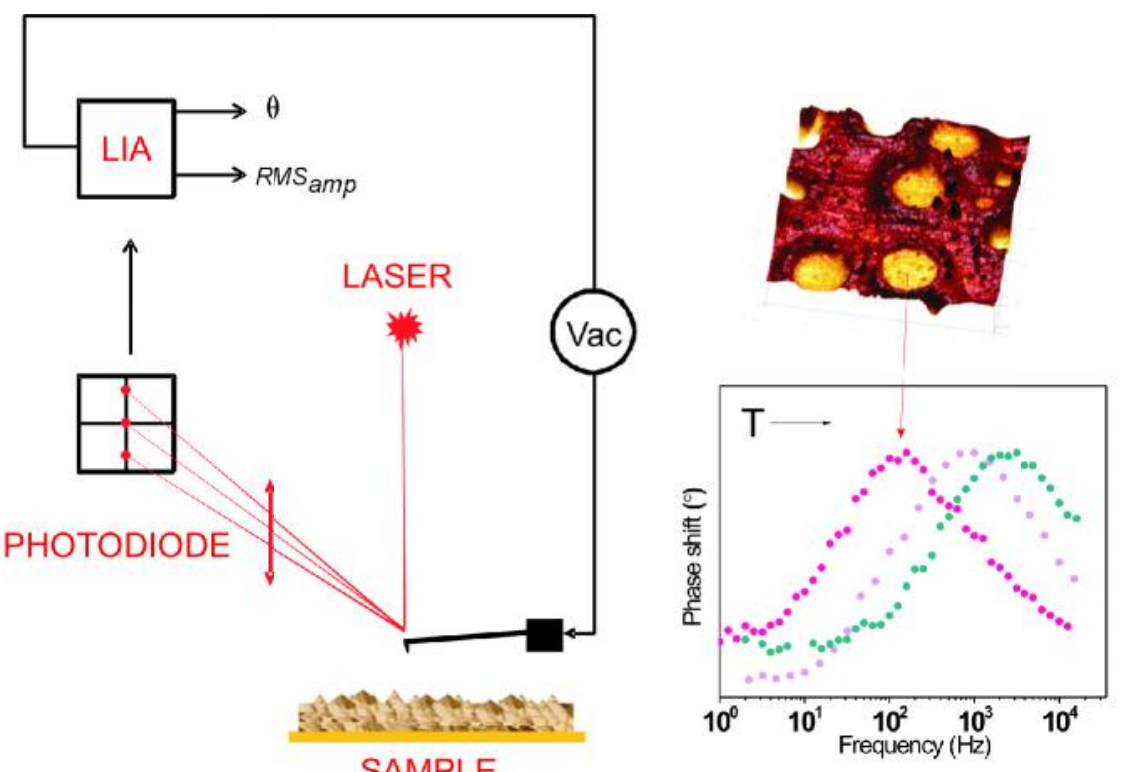

Figure 1. AM-EFM implementation scheme. The cantilever oscillation is detected by the photodiode. The signal with frequency double than that of the applied AC voltage is filtered by the LIA and both, amplitude and phase, are used to obtain either images or spectra of the dielectric response. In particular, the output shown in the Figure corresponds to a PVAc/PS (25/75 wt \%) sample. 


\section{EXPERIMENTAL DETAILS}

\subsection{Samples}

Polymer films of pure PVAc $(\mathrm{Mw}=83000)$ were prepared by spin-coating polymer-toluene solutions on gold sputtered glass (in turn mounted on metallic disks of $12 \mathrm{~mm}$ diameter). Thicknesses of about 200-250 $\mathrm{nm}$ were obtained by using a $4 \mathrm{wt} \%$ PVAc/Toluene solution. PS (Mw=73950)/PVAc samples (25/75 wt \% and 75/25 wt \%) were prepared by using the same procedure. These thicknesses were chosen so as to obtain dielectric responses free of any size effects [4].

2.2 Broadband Dielectric Spectroscopy (BDS): Broadband dielectric spectroscopic measurements were performed on disc shaped samples with a diameter of $20 \mathrm{~mm}$ and a thickness of about $0.1 \mathrm{~mm}$. Broadband high-resolution dielectric spectrometer (Novocontrol Alpha) was used to measure the complex dielectric permittivity in the frequency range from $10^{-2}$ to $10^{6} \mathrm{~Hz}$. Isochronal temperature curves were obtained at 50 $\mathrm{kHz}$ and $215 \mathrm{kHz}$ from the corresponding isothermal experiments. The sample temperature was controlled by nitrogen gas flow that provides temperature stability of about $\pm 0.1 \mathrm{~K}$.

\subsection{AFM general setup}

Topography and mechanical phase images were recorded simultaneously in moderate tapping mode, with an Atomic Force Microscope MultiMode 8 (Bruker). The measurements were performed using Antimony $(\mathrm{Sb})$ doped Si cantilevers, coated with $\mathrm{Pt} / \mathrm{Ir}$ (SCM-PIT Bruker). The nominal values for the natural frequency $\left(f_{0}\right)$, tip radius $(R)$, cantilever spring constant $(k)$ and sensitivity $(\chi)$ for the probes are $75 \mathrm{kHz}, 20$ $\mathrm{nm}, 1.5-3 \mathrm{~N} / \mathrm{m}$ and $20 \mathrm{mV} / \mathrm{nm}$, respectively. Additionally, for the analysis of the electrical excitation of higher eigenmodes, softer (SCM-PIC) probes were also used ( $f_{o}$ $=10 \mathrm{kHz}, \mathrm{R}=20 \mathrm{~nm}$ and $k=0.2 \mathrm{~N} / \mathrm{m}$ ). In this case the electrical measurements were performed by setting a constant tip-sample distance (measured from the starting contact position) by using the generic sweep mode of the software (Bruker). Both electrical excitation and filtering of the resulting photodiode signal were then performed with the external LIA. 
The temperature of the sample was controlled (from room temperature up to $150^{\circ} \mathrm{C}$ ) by using a Thermal Applications Controller (TAC, Bruker). A silicone cap was used as sealing to improve the thermal stabilization of the system. The atmosphere inside the silicon cap was controlled by using a dry nitrogen flow. Two different external Lock-in Amplifiers were employed in the electrical experiments: Stanford Research SR830 (low frequency range up to $100 \mathrm{kHz}$ ) and Princeton Applied Research 5302 (High frequency range up to $1 \mathrm{MHz}$ ).

\section{$2.4 n \mathrm{DI}$}

The images of the materials were acquired in moderate tapping mode by using SCM-PIT tips and either $1 \mathrm{~V}$ or $5 \mathrm{~V}$ as excitation voltages. Both the scan rate and the LIA time constants were properly selected in order to prevent undersampling.

\section{$2.5 n \mathrm{DS}$}

Both electric phase and RMS amplitude were recorded using a homemade Labview routine, at excitation voltages of either $1 \mathrm{~V}$ or $5 \mathrm{~V}$ (according to the experiment) and typical tip-sample distances of $25 \mathrm{~nm}$. As previously mentioned, a reference spectrum was acquired by using gold as substrate. Dielectric spectra were obtained by evaluating the phase shift (reference - sample response) as a function of electrical excitation frequency.

\section{RESULTS AND DISCUSSION}

\subsection{Analysis of the electrical excitation of the cantilever second eigenmode}

In order to explore the possibility of using higher eigenmodes to perform $n \mathrm{DS}$, as a first step we measured the cantilever response for different electrical excitation frequencies (no piezo excitation). Figure 2 shows the RMS oscillation amplitude obtained at $2 f_{e}$ (as shown in equation 2, at this frequency the desired electrical information appears independently of surface potential and $V_{D C}$ ). The experiments were performed over a gold substrate at room temperature by applying an excitation voltage of $5 \mathrm{~V}$. This substrate was chosen so as to obtain a dissipation free sample, in order to exclusively analyze the cantilever response. As shown, $\left|R M S_{a m p}\right|$ is nearly constant in the $1-10^{4}$ 
$\mathrm{Hz}$ range, where the fundamental mode is responsible for the cantilever oscillation amplitude. A maximum in the oscillation amplitude value appears at $35 \mathrm{kHz}$, indicating the presence of the mechanical resonance (fundamental mode). At this point it is worth stressing that the electrical excitation of the cantilever at $f_{e}$ produces an output with three components (see equation 1): a DC component, and two frequency dependent components, at $f_{e}$ and $2 f_{e}$. As $n \mathrm{DS}$ records the response at $2 f_{e}$, the fundamental mode appears at $f_{e}=35 \mathrm{kHz}$ in Figure 2 spectra.

As expected, the oscillation amplitude rapidly decreases for higher excitation frequencies, reaching its minimum at about $10^{5} \mathrm{~Hz}$. At higher frequencies it increases again, reaching at about $220 \mathrm{kHz}$ a second maximum in the cantilever oscillation amplitude (second eigenmode). The same experiments were performed by using a cantilever with lower resonance frequency (lower $f_{0}$ ) as shown in the Figure 2 inset. As it is clear from the figure, the obtained results are similar for both probes.

These results show that it is possible for us to detect electrical forces at higher frequencies than $f_{e}=f_{0} / 2$ by exciting the cantilever second eigenmode, as suggested by Martin et al. [28]. In addition, it is worth noticing that the presence of harmonics of the first mode was not detected in our experiments. As the harmonics are produced by the non-linear interactions between the tip and the sample [12], this result could suggest that interaction strength is low due to the relatively high tip-sample distances employed [29]

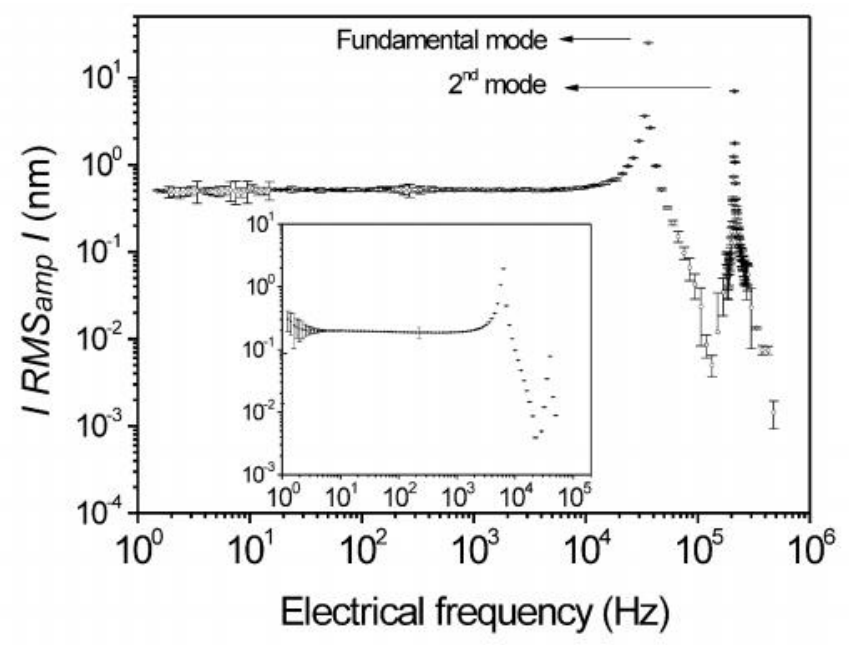


Figure 2 RMS oscillation amplitude $\left(2 f_{e}\right)$ of SCM - PIT tip Bruker as a function of applied electrical excitation frequency, $f_{e}$. The measurements were performed over a gold substrate at room temperature, at a tip-sample distance of $25 \mathrm{~nm}$ and by applying a sinusoidal voltage of frequency $f_{e}$ and $5 \mathrm{~V}$ amplitude. Inset: SCM - PIC tip Bruker, resonance frequency $10 \mathrm{kHz}$.

\subsection{Analysis of the signal-to-noise ratio}

In this section we aim to evaluate the noise level on $n \mathrm{DS}$ measurements while performed in extended frequency range. In a first step, we studied the effect of thermal noise on the cantilever oscillation amplitude. In a second step, we evaluated the statistical influence of the signal-to-noise ratio along the extended frequency range.

\subsubsection{Thermal noise}

As a first step, the cantilever oscillations produced by thermal noise were measured (neither piezo nor electric driving forces were applied). The experiments were performed at room temperature, without the silicone cap sealing (in order to detect the maximum noise level). The raw thermal oscillations were recorded by using the High Speed Data Capture ${ }^{\mathrm{TM}}$ software of the microscope (Bruker). As shown in Figure 3, the raw thermal noise oscillation amplitude shows typical values according to the spring constant of the cantilever and the temperature of the experiment [30]. In this way, the noise is about two orders of magnitude lower than the corresponding $2 f_{e}$ cantilever oscillations produced by the electrical excitation (see Figure 2). 

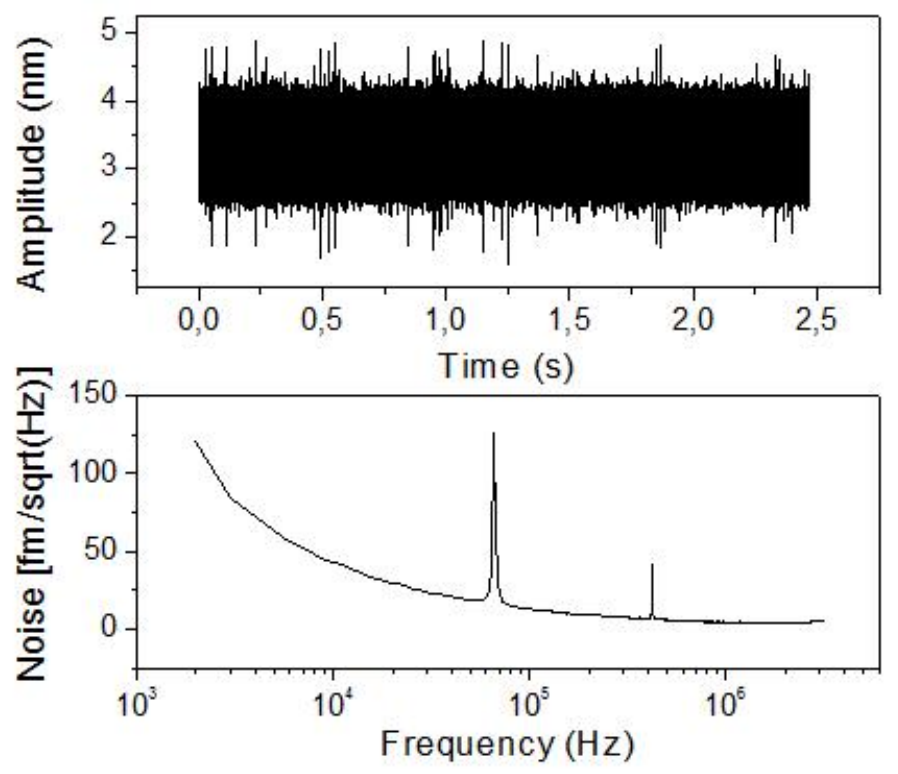

Figure 3 Thermal noise amplitude (time and frequency domain) acquired by using the High Speed Data Capture ${ }^{\mathrm{TM}}$ software of the microscope. The measurements were performed on a grounded gold substrate at room temperature, without silicone cap sealing.

\subsubsection{Accuracy of the $n \mathrm{DS}$ measurements.}

As the signal-to-noise ratio changes significantly along the extended frequency range (see error bars in Figure 2 and thermal noise in Figure 3), we estimated the statistical deviations obtained at two different cantilever oscillation amplitudes: $A_{1}$ (typical amplitude detected for electrical excitations at frequencies lower than the resonance frequency of the fundamental mode) and $A_{2}$ (typical amplitude detected in the range of frequencies between first and second eigenmode resonances). In order to attain the desired values $A_{1}(=0.5 \mathrm{~nm})$ and $A_{2}(=0.02 \mathrm{~nm})$ the AC $(1 \mathrm{kHz})$ excitation voltage amplitude was set to $5 \mathrm{~V}$ and $1 \mathrm{~V}$, respectively (changing the excitation voltage at a constant frequency allows an accurate control of the cantilever oscillation amplitude, which as shown in equation 3 scales as $\mathrm{V}_{0}^{2}$ ). Both $\left|R M S_{a m p}\right|$ and $\theta$ (at different locations of a grounded gold substrate) were measured. Representative results obtained for a SCM-PIT probe are presented in Figure 4. As expected, the associated dispersion for $\theta$ measurements performed using $A_{l}$ is substantially lower than the one obtained when measuring with $A_{2}$. Even in this worst situation the dispersion of the data is lower 
than 0.5 degrees.
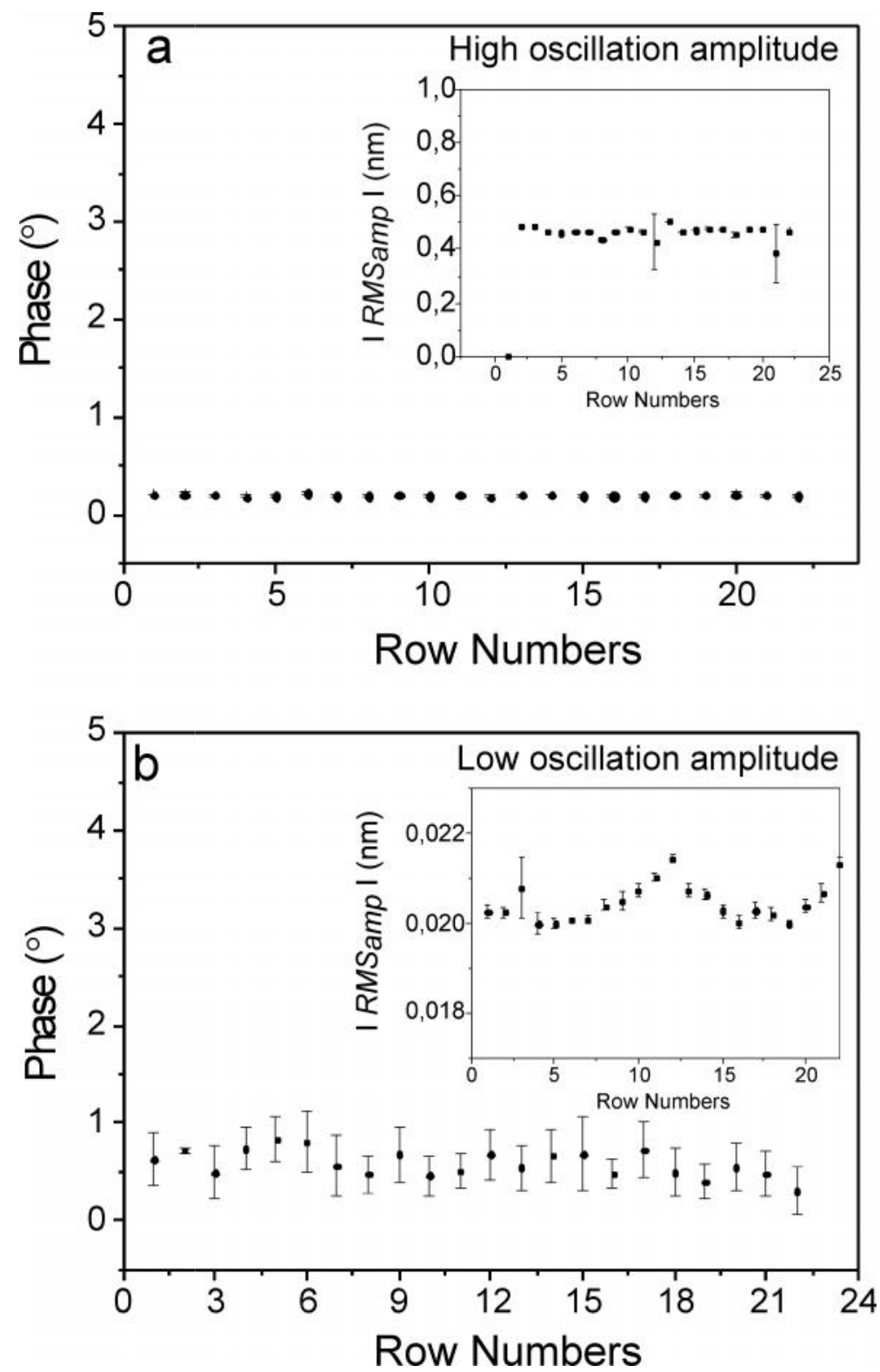

Figure 4 Dielectric phase measurements on a grounded gold substrate at an excitation frequency of $1 \mathrm{kHz}$. a) $5 \mathrm{~V}$ (cantilever oscillation amplitude values of about $0.5 \mathrm{~nm}$ ). b) $1 \mathrm{~V}$ (cantilever oscillation amplitude values of about $0.02 \mathrm{~nm}$ ). Each point of the graph represents a different location of the sample, where about 60 values were acquired and averaged.

\subsection{Studying the dipolar relaxations by using the cantilever second eigenmode}

As a final step, a well documented dissipative process was studied by using both the 
fundamental and the second eigenmodes. Proof of concept experiments were performed on thin film polymer blends of polyvinyl acetate, PVAc (a polymer showing a prominent dielectric relaxation) and polystyrene, PS (which do not show detectable dielectric relaxation in the explored range). The PVAc/PS system is immiscible and the blends show phase separation, with islands of the minority component spread in a matrix of the other one.

\subsection{1 $n \mathrm{DI}$}

In a first series of experiments, imaging at a fixed frequency and different temperatures was performed by detecting the phase variations of the $2 f_{e}$ component of the electrical force along the sample. Figure $5\left(f_{e}=50 \mathrm{kHz}\right)$ shows that the electrical phase contrast is weak at low and high temperatures, but passes through a maximum at intermediate temperatures (showing the areas where PVAc molecular mobility takes place with a time scale in the range of the reciprocal of the applied electric voltage frequency [8]).
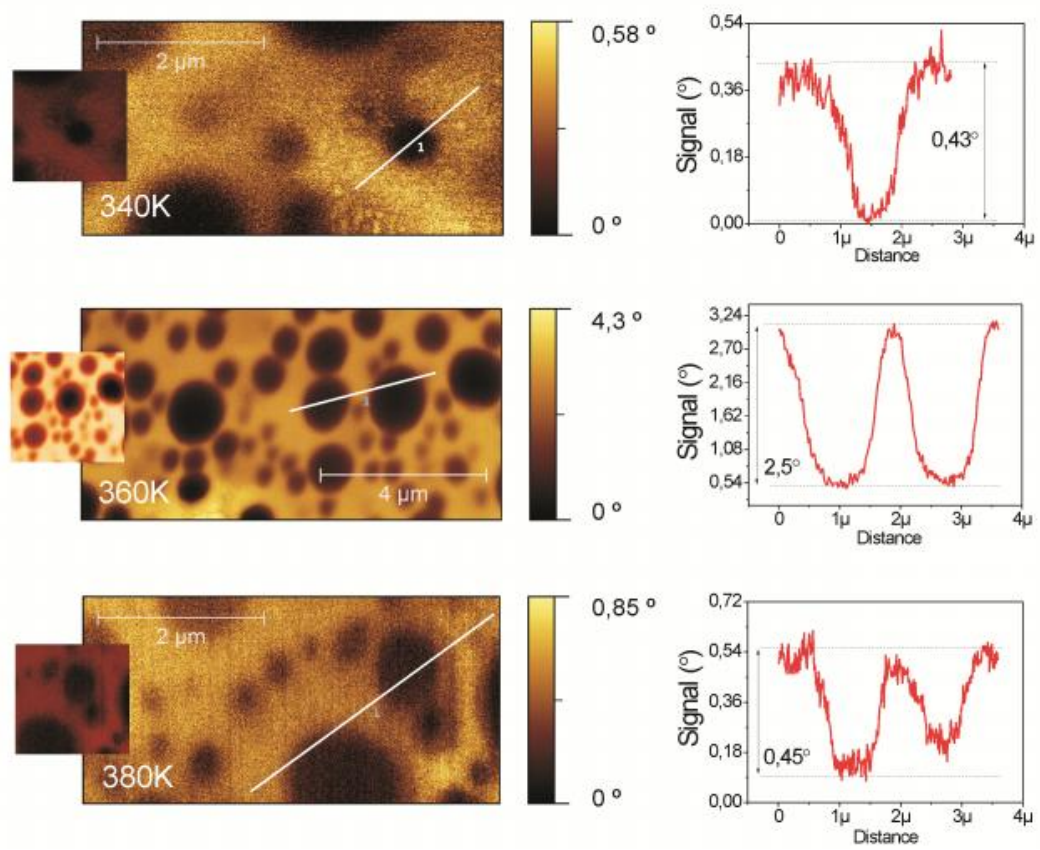

Figure 5 Electrical phase images of a PS/PVAc sample (25/75 wt \%) obtained at different temperatures and at $50 \mathrm{kHz}$. Brighter areas correspond to higher losses, indicative of the segmental relaxation of PVAc. The profiles were obtained along the line " 1 " of each image, and show the dielectric dissipation contrast between the two phases. Insets: dissipation contrast in the same scale $\left(0-3.7^{\circ}\right)$. 
Figure 6 shows the dielectric losses $\left(\varepsilon^{\prime \prime}\right)$ temperature dependence for a pure PVAc sample, measured by standard BDS at $50 \mathrm{kHz}$ and $215 \mathrm{kHz}$ (isochrones). Electrical phase contrast images obtained through $n \mathrm{DI}$ at the same frequency were added in order to show that the direct relationship between the image contrast and dielectric relaxation still holds at these higher frequencies.
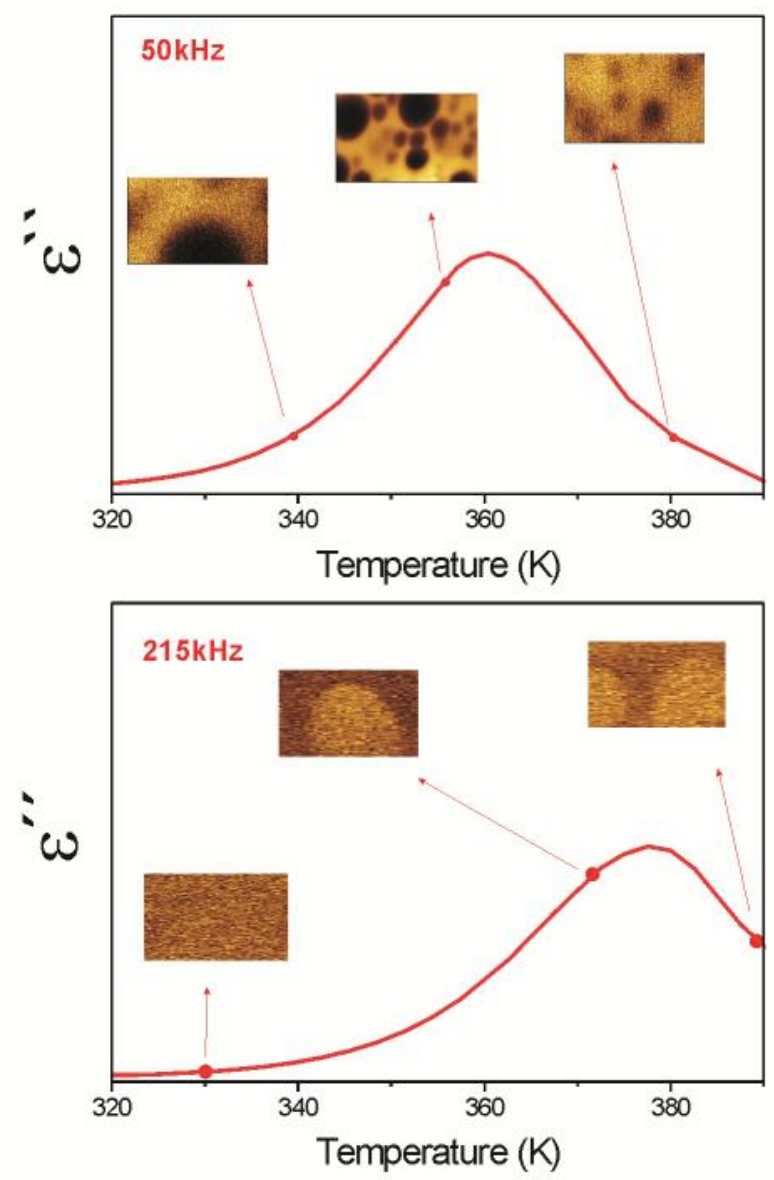

Figure $6 \varepsilon^{\prime \prime}$ isochrones (obtained by BDS at $50 \mathrm{kHz}$ and $215 \mathrm{kHz}$ ) for a pure PVAc sample. Insets: electrical phase images showing the electrical phase contrast (PS/PVAc $25 / 75 \%$ at $50 \mathrm{kHz}$ and PS/PVAc $75 / 25 \%$ at $215 \mathrm{kHz}$ ) at each temperature.

In Figure 7, the image contrast is quantitatively analyzed. It is found that the maximum contrast at these frequencies, where the second eigenmode dominates, is about two orders of magnitude lower than that obtained by using the fundamental mode. This 
contrast reduction could be related to the lower detected signal, i.e. phase contrast between phases is damped by noise signal. The image contrast alone does not render information about the in-depth composition; it simply shows that there is a higher/lower concentration of the relaxing component. On the contrary, the intensity of the measured phase shift peak is directly related with the amount of relaxing species within the probe volume.

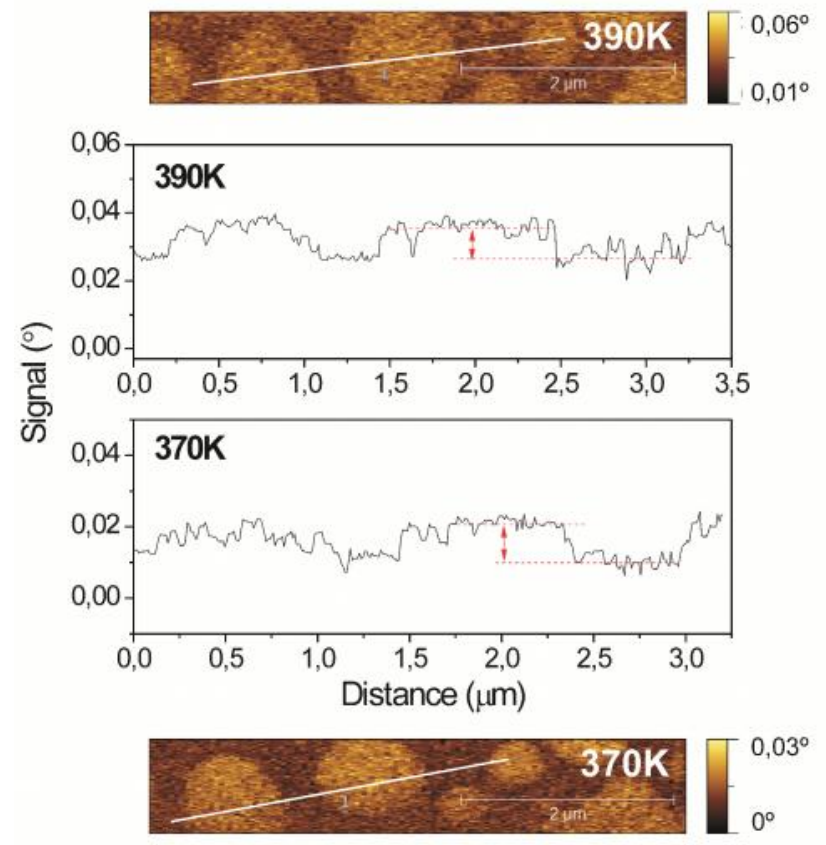

Figure 7 Electrical phase images of a PS/PVAc sample (75/25 wt \%) obtained at different temperatures and at $215 \mathrm{kHz}$ ( $2^{\text {nd }}$ eigenmode). Brighter areas correspond to higher losses, indicative of the segmental relaxation of PVAc. The profiles were obtained along the line " 1 " of each image.

\subsection{2 $n \mathrm{DS}$}

In a second series of experiments, the dielectric relaxation of a pure PVAc sample was measured by $n$ DS sweeping in frequency at 335 and 343K. Figure 8 shows the obtained frequency dependent phase shift (the zero phase was determined by making a similar experiment on a conductive gold film) for a SCM-PIT tip $\left(f_{o}=70 \mathrm{kHz}\right)$. It is clear that at these temperatures segmental motions in PVAc occur at a rate matching well the accessible frequency window, as evidenced by the detected peaks.

The higher dispersion of the points at high frequencies (grey area in Figure 8) reflects 
the influence of the lower oscillation amplitudes (and therefore, the previously observed lower signal-to-noise ratio). In addition, increasing the temperature also increases the thermal drift, which in turn affects the statistics of the measurements over the whole range. It is clear that for some frequencies (around $200 \mathrm{kHz}$ in Figure 8), the oscillation amplitude is too low to be detected. It is worth stressing that the PS signal cannot be detected due to its low dielectric strength [31].

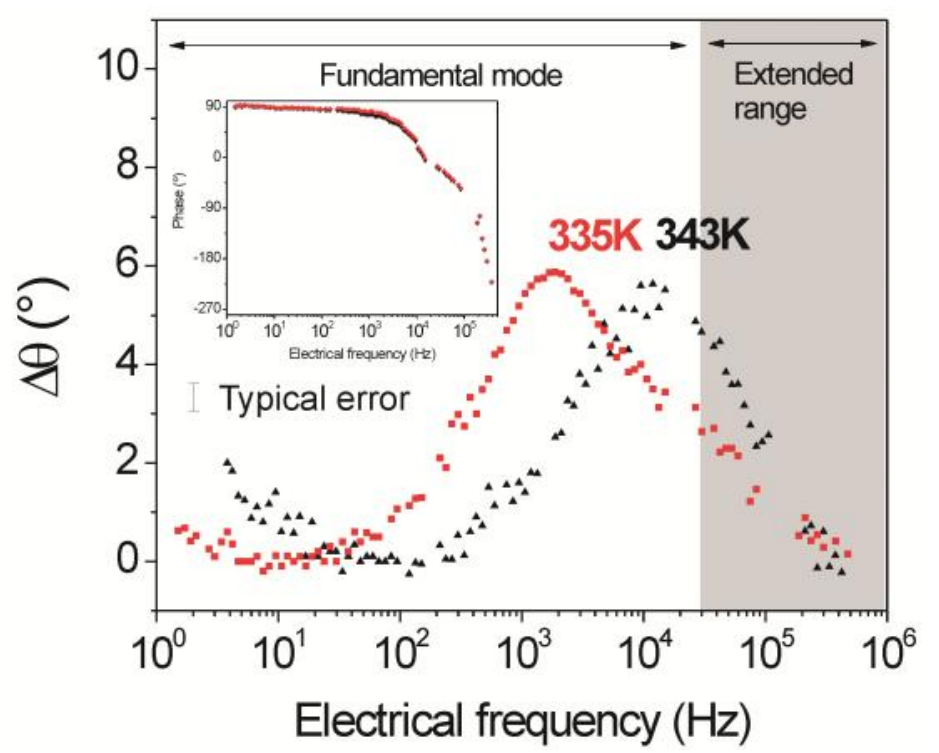

Figure $8 n$ DS phase shift spectra of a pure PVAc sample. A SCM-PIT probe was employed, and the measurements were performed at 335 and 343K. Inset: raw phase values of reference (red) and PVAc sample (black) at $335 \mathrm{~K}$.

Thus, these experiments have shown that it is possible to use higher eigenmodes of the cantilever for detecting dielectric dissipative processes at frequencies above $f_{0}$. As a result, the $n \mathrm{DS}$ frequency window has been extended up to nearly $0.5 \mathrm{MHz}$.

\section{CONCLUSIONS}

A detailed experimental analysis of the electrical excitation of the second eigenmode of a conductive cantilever has been presented. As a result, by using this second eigenmode response, the experimental frequency range of nanoDielectric Spectroscopy has been 
extended towards higher frequencies by about 2 decades. In addition, the dispersion of the measurements performed in the extended frequency range has also been analyzed. The experimental uncertainty has been found to be higher in comparison with that of the fundamental mode, but the data still present a good signal-to-noise ratio. Finally, dielectric loss processes have been studied on model polymer samples by combining both fundamental and second eigenmodes of the cantilever. The obtained $n \mathrm{DS}$ spectra and images have shown that the cantilever oscillations can probe the dipolar relaxations within this broader frequency range.

\section{ACKNOWLEDGMENTS}

We acknowledge the financial support provided by the Basque Country Government (IT-654-13) and the Spanish Ministry of Science and Innovation (MAT2012-31088). Financial support from EU-funded 'European soft matter infrastructure' (Reference 262348 ESMI) is also acknowledged. 


\section{REFERENCES}

[1] F. Kremer, A. Schonhals, Broadband Dielectric Spectroscopy, Springer-Verlag, New York, 2003.

[2] P.S. Crider, M.R. Majewski, J. Zhang, H. Oukris, N.E. Israeloff, Local dielectric spectroscopy of polymer films, Applied Physics Letters, 91 (2007) 013102-013103.

[3] M. Labardi, D. Prevosto, K.H. Nguyen, S. Capaccioli, M. Lucchesi, P. Rolla, Local dielectric spectroscopy of nanocomposite materials interfaces, in, AVS, 2010, pp. C4D11-C14D17.

[4] H.K. Nguyen, D. Prevosto, M. Labardi, S. Capaccioli, M. Lucchesi, P. Rolla, Effect of Confinement on Structural Relaxation in Ultrathin Polymer Films Investigated by Local Dielectric Spectroscopy, Macromolecules, 44 (2011) 6588-6593.

[5] H.K. Nguyen, M. Labardi, S. Capaccioli, M. Lucchesi, P. Rolla, D. Prevosto, Interfacial and Annealing Effects on Primary $\alpha$-Relaxation of Ultrathin Polymer Films Investigated at Nanoscale, Macromolecules, 45 (2012) 2138-2144.

[6] C. Riedel, R. Arinero, P. Tordjeman, M. Ramonda, G. Lévêque, G.A. Schwartz, D.G. De Oteyza, A. Alegria, J. Colmenero, Determination of the nanoscale dielectric constant by means of a double pass method using electrostatic force microscopy, J. Appl. Phys., 106 (2009) 024315-024315-024316.

[7] C. Riedel, R. Arinero, P. Tordjeman, G. Lévêque, G.A. Schwartz, A. Alegría, J. Colmenero, Nanodielectric mapping of a model polystyrene-poly(vinyl acetate) blend by electrostatic force microscopy, Phys. Rev. E, 81 (2010) 010801.

[8] C. Riedel, R. Sweeney, N.E. Israeloff, G.A. Schwartz, R. Arinero, P. Tordjeman, G. Lévêque, A. Alegría, J. Colmenero, Imaging dielectric relaxation in nanostructured polymers by frequency modulation electrostatic force microscopy, Appl. Phys. Lett., 96 (2010) 213110.

[9] C. Riedel, A. Alegria, G.A. Schwartz, R. Arinero, J. Colmenero, J.J. Saenz, On the use of electrostatic force microscopy as a quantitative subsurface characterization technique: A numerical study, Applied Physics Letters, 99 (2011) 023101-023103.

[10] G.A. Schwartz, C. Riedel, R. Arinero, P. Tordjeman, A. Alegría, J. Colmenero, Broadband nanodielectric spectroscopy by means of amplitude modulation electrostatic force microscopy (AM-EFM), Ultramicroscopy, 111 (2011) 1366-1369.

[11] C. Riedel, A. Alegría, G.A. Schwartz, J. Colmenero, J.J. Sáenz, Numerical study of the lateral resolution in electrostatic force microscopy for dielectric samples, Nanotechnology, 22 (2011) 285705.

[12] R. García, Amplitude modulation Atomic Force Microscopy, WILEY-VCH, Weinheim, 2010.

[13] R. Garcia, R. Proksch, Nanomechanical mapping of soft matter by bimodal force microscopy, European Polymer Journal, (2013).

[14] T.R. Rodriguez, R. Garcia, Compositional mapping of surfaces in atomic force microscopy by excitation of the second normal mode of the microcantilever, Applied Physics Letters, 84 (2004) 449-451.

[15] W.S. Robert, N. Nicola, S. Andreas, Multifrequency electrostatic force microscopy in the repulsive regime, Nanotechnology, 18 (2007) 065502.

[16] N.F. Martínez, J.R. Lozano, E.T. Herruzo, F. Garcia, C. Richter, T. Sulzbach, R. Garcia, Bimodal atomic force microscopy imaging of isolated antibodies in air and liquids, Nanotechnology, 19 (2008) 384011.

[17] R. Proksch, Multifrequency, repulsive-mode amplitude-modulated atomic force microscopy, Applied Physics Letters, 89 (2006) 113121-113123. 
[18] M. Baumann, R.W. Stark, Dual frequency atomic force microscopy on charged surfaces, Ultramicroscopy, 110 (2010) 578-581.

[19] R. Garcia, R. Pérez, Dynamic atomic force microscopy methods, Surface Science Reports, 47 (2002) 197-301.

[20] A. Gil, J. Colchero, J. Gómez-Herrero, A.M. Baró, Electrostatic force gradient signal: resolution enhancement in electrostatic force microscopy and improved Kelvin probe microscopy, Nanotechnology, 14 (2003) 332.

[21] G.M. Sacha, J.J. Saenz, Cantilever effects on electrostatic force gradient microscopy, Applied Physics Letters, 85 (2004) 2610-2612.

[22] L. Fumagalli, G. Ferrari, M. Sampietro, G. Gomila, Dielectric-constant measurement of thin insulating films at low frequency by nanoscale capacitance microscopy, Applied Physics Letters, 91 (2007) 243110-243113.

[23] G. Gomila, J. Toset, L. Fumagalli, Nanoscale capacitance microscopy of thin dielectric films, J. Appl. Phys, 104 (2008) 024315.

[24] L. Fumagalli, G. Ferrari, M. Sampietro, G. Gomila, Quantitative Nanoscale Dielectric Microscopy of Single-Layer Supported Biomembranes, Nano Letters, 9 (2009) 1604-1608.

[25] L. Fumagalli, G. Gramse, D. Esteban-Ferrer, M.A. Edwards, G. Gomila, Quantifying the dielectric constant of thick insulators using electrostatic force microscopy, Applied Physics Letters, 96 (2010) 183107-183103.

[26] S. Magonov, J. Alexander, Single-pass Kelvin force microscopy and dC/dZ measurements in the intermittent contact: applications to polymer materials, Beilstein Journal of Nanotechnology, 2 (2011) 15-27.

[27] M.M. Kummali, G.A. Schwartz, A. Alegria, J. Colmenero, Single vs double pass EFM imaging, Imaging \& Microscopy, 1 (2012) 43-45.

[28] Y. Martin, D.W. Abraham, H.K. Wickramasinghe, High-resolution capacitance measurement and potentiometry by force microscopy, Applied Physics Letters, 52 (1988) 1103-1105.

[29] R. Hillenbrand, M. Stark, R. Guckenberger, Higher-harmonics generation in tapping-mode atomic-force microscopy: Insights into the tip-sample interaction, Applied Physics Letters, 76 (2000) 3478-3480.

[30] B. Hans-Jurgen, J. Manfred, Calculation of thermal noise in atomic force microscopy, Nanotechnology, 6 (1995) 1-7.

[31] L.A. Miccio, M.M. Kummali, G.A. Schwartz, A. Alegría, J. Colmenero. Dielectric spectroscopy at the nanoscale by atomic force microscopy: A simple model linking materials properties and experimental response, J. Appl. Phys., 115 (2014), 184305-184315. 